\title{
Tarification et Interfinancement dans les Régimes Obligatoires d'Assurance-Automobile
}

\author{
par Claude Fluet*
}

\section{Introduction}

Le principe de l'assurance étant la mutualisation des risques, on considère qu'il y a interfinancement à l'intérieur d'un regroupement d'assurés lorsque les primes d'assurances ne sont pas actuarielles, c'est-à-dire lorsqu'elles diffèrent de l'espérance mathématique de l'indemnité que recevra un assuré en cas de sinistre'. Avec des primes actuarielles, chaque assuré assume le coût qu'il impose à la collectivité des assurés. Outre la question d'équité, une telle tarification peut se justifier pour des raisons d'efficacité: lorsque chacun est libre de déterminer sa couverture d'assurance, une prime actuarielle incite à choisir une couverture optimale; de plus, lorsque le risque dépend du comportement de l'assuré, la variation de la prime en fonction du risque contribue à un choix approprié d'activités de prévention. Un marché concurrentiel d'assurance tendra à établir des primes proportionnées aux risques individuels, bien qu'une certaine mesure d'interfinancement soit possible dans un groupe s'il y a information imparfaite sur les risques que chaque assuré représente. Les exemples les plus fréquents d'interfinancement s'observent toutefois dans certains régimes publics obligatoires d'assurance à couverture prédéterminée. Le propos de cet article est de discuter, dans le contexte particulier de l'assurance automobile, de l'interfinancement qu'implique une tarification uniforme comme celle que pratique le régime public d'assurance automobile no-fault du Québec. Le cadre analytique développé ici permet cependant de discuter également de formules de tarification différentes qui sont ou pourraient être utilisées dans d'autres systèmes obligatoires d'assurance automobile² .

\footnotetext{
*Université du Québec à Montréal.

Je tiens à remercier Eric Briys, Denis Kessler, Bernhard Kromschröder, Pierre Lefebvre, Jean-François Outreville, Pierre Mohnen, ainsi qu'un lecteur anonyme, pour leurs commentaires sur une première version de ce texte. Source de financement: PAFACC (UQAM). Ce texte, dont les conclusions n'engagent que l'auteur, constitue le prolongement d'une recherche subventionnée par la Régie de l'assurance automobile du Québec.

${ }^{1}$ On suppose ici que les coûts d'administration de l'assurance sont nuls et que les gains liés à la diversification des risques sont pleinement exploites.

2 Les effets redistributifs d'une tarification uniforme en assurance ont été analysés par Pauly [1970], [1974] et Johnson [1977]. La possibilité, lorsqu'il y a anti-sélection, d'équilibres mélangeants avec interfinancement dans un marché concurrentiel d'assurance a été démontrée initialement par Wilson [1977]. Voir Hoy [1982] pour une application des résultats de Wilson à l'analyse des méthodes traditionnelles de classification des risques et Dahlby [1983] pour une étude empirique de ces questions en assurance automobile.
} 
Du régime québécois on ne retiendra que les éléments suivants: (i) toutes les victimes de dommages corporels résultant d'accidents de la route sont indemnisées sans égard à la responsabilité de quiconque; (ii) le régime vise avant tout la compensation des pertes économiques, notamment la perte de revenu de travail pour cause d'incapacité ou la perte de soutien financier pour les personnes à charge dans le cas d'un décès; (iii) la totalité du coût de l'assurance est facturée aux propriétaires ou conducteurs de véhicules. Pour l'essentiel, surtout si on se limite aux voitures de tourisme qui constituent la plus grande partie du parc automobile, le financement repose sur des primes uniformes ${ }^{3}$. Ce mode de financement est évidemment facilité par le fait que le régime est administré par une société d'Etat. D'autres formules de tarification seraient néanmoins possibles tout en conservant le principe de l'assurance obligatoire et de l'indemnisation des victimes sans égard à la responsabilité:

(i) Selon la formule de l'assurance individuelle, les primes seraient proportionnées à la probabilité de réclamation du couple véhicule-conducteurs; elles correspondraient ainsi au coût actuariel des dommages auxquels s'exposent le conducteur et ses passagers, y inclus les dommages occasionnés aux tiers hors véhicules. C'est une formule de ce genre qu'on trouve généralement dans les systèmes no-fault administrés par le secteur privé (voir Tunc [1981a] pour une description de certains de ces régimes). Ceci inclut évidemment les tarifications bonus-malus lorsque celles-ci sont utilisées pour identifier la probabilité de réclamation d'un conducteur, quand celle-ci n'est pas parfaitement connue a priori (cf. Henriet et Rochet [1986]).

(ii) Bien que l'indemnisation soit no-fault, la facturation du coût de l'assurance pourrait faire appel au principe de responsabilité, ce qui peut être fait de façon plus ou moins stylisée en utilisant par exemple des schémas de circonstances d'accidents pour l'imputation de la responsabilité; la prime du couple véhicule-conducteurs serait dans ce cas égale au coût actuariel des dommages qu'il est susceptible de subir du fait de sa propre «faute» et de ceux qu'il est susceptible de «causer» aux tiers automobilistes. Une formule semblable, qui fait appel au principe de l'indemnisation des victimes sans égard à la responsabilité tout en retenant le principe de responsabilité pour la facturation du coût de l'assurance (et comme incitation à la prudence), a été envisagée dans plusieurs projets de modification aux lois sur l'assurance automobile (voir par exemple le Report by the Automobile Accident Committee of British Columbia [1983]).

Il ressort de ceci que l'appréciation du degré d'interfinancement associé à une tarification uniforme dépend du cadre retenu pour l'imputation du coût des accidents. On verra plus loin qu'aucune des formules que l'on vient de décrire n'est véritablement appropriée, chacune impliquant une forme d'interfinancement. En termes succincts, l'argumentation présentée dans les prochaines sections est la suivante. Indépendamment de toute considération relative à l'incitation à la prudence, on s'attend à ce qu'il y ait interfinancement avec des primes uniformes, par exemple à ce que les «bons risques» en termes de fréquence d'accidents subventionnent les «mauvais risques». Comme les indemnités versées (donc le montant assuré en cas d'accident) sont aussi très différentes d'une catégorie d'automobilistes à

\footnotetext{
${ }^{3}$ Les primes sont perçues sur les permis de conduire et sur les véhicules et peuvent varier selon la classe de permis et le type de véhicule (voitures de tourisme, véhicules utilitaires selon le poids et la fonction, etc.), indépendamment des caractéristiques de risque ou du dossier d'accident du conducteur ou du propriétaire, sauf pour des ajustements mineurs. Pour une brève description du régime québécois, voir Boyer et Dionne [1987].
} 
l'autre, une évaluation simple du degré d'interfinancement consisterait à calculer le coût actuariel de différentes catégories de véhicules-conducteurs, compte tenu de leur probabilité d'accident et du dommage subi ${ }^{4}$. Mais s'il va de soi que l'addition d'un mauvais risque au bassin d'automobilistes crée une externalité pécuniaire avec une tarification uniforme (puisqu'elle augmentera la prime de tous les assurés), il est clair aussi que l'usage de l'automobile est un exemple parfait d'externalité «technologique». L'interfinancement devrait donc s'évaluer dans un contexte d'interaction directe, le coût que représente un mauvais risque incluant l'effet que l'addition d'un tel risque aura sur la probabilité d'accident des autres automobilistes (ce qui jouera quel que soit le mode de tarification). On sait que le principe de responsabilité et l'assurance de responsabilité qui en découle sont une façon d'imputer à un conducteur le coût qu'il impose aux autres par les dommages qu'il est susceptible de causer. Ceci suggère par conséquent que l'on mesure l'interfinancement en tenant compte du coût actuariel des dommages (à lui-même et à autrui) dont la responsablité pourrait être imputée à chaque conducteur. Cependant, cette seconde approche n'est à son tour pas exempte d'ambiguité: il y a, en effet, une symétrie entre la tarification sur base d'assurance individuelle (chacun assumant le coût actuariel des dommages auquels il s'expose, quelle qu'en soit la cause) et la tarification sur base d'assurance de responsabilité (chacun assumant le coût actuariel des dommages qu'il est susceptible de causer). Dans le premier cas, l'addition d'un «mauvais conducteur», par son effet sur la probabilité d'accident des autres automobilistes, entraîne pour ces derniers une hausse des primes d'assurance individuelle. De façon symétrique, dans une tarification sur base de responsabilité, l'addition d'un conducteur à perte élevée en cas d'accident se traduit par une hausse du coût moyen de l'assurance-responsabilité. Autrement dit, ce conducteur (même s'il est prudent) impose aux autres conducteurs le coût actuariel des dommages que ceux-ci sont susceptibles de lui causer.

Il est évident, par conséquent, qu'on ne peut discuter d'interfinancement en assurance automobile sans que soient déterminés au préalable les droits des conducteurs sur la ressource commune que représente le réseau routier, puisque la décision de conduire (d'accéder au réseau) va de pair ici avec la participation obligatoire au régime d'assurance. En d'autres termes, cette question peut se traiter comme un problème classique de financement d'un bien public sujet à congestion; le coût en accidents représente alors une partie du coût social d'utilisation de ce bien, les primes d'assurance étant assimilées à des péages individualisés pour différentes catégories d'utilisateurs. Dans ce qui suit, on supposera que le régime d'assurance n'a à subventionner aucune catégorie d'automobilistes. Un conducteur se verra reconnaître un droit d'accès au réseau dans la mesure où il est prêt à payer le coût qu'implique sa décision d'y accéder. On obtient naturellement de cette façon une

\footnotetext{
${ }^{4}$ Dans le régime québécois, les indemnités accordées à une victime sont fonction de la perte de revenu de travail et du nombre de personnes à charge. En raison du facteur âge, il y a ainsi une corrélation négative entre la probabilité d'accident du conducteur et le remboursement qui lui est effectivement assuré. Par exemple, la fréquence d'accidents mortels chez les conducteurs dans le groupe d'âge des 20-24 ans était en 1983 de plus du double de celle des 45-54 ans. Pour la même année, des données internes de la Régie de l'assurance automobile du Québec montrent cependant que le coût capitalisé moyen d'un décès chez les $20-24$ ans représente environ $20 \%$ du coût d'un décès chez les $45-54$ ans. Pour les accidents avec blessures seulement, les différences de coût entre les catégories d'âge sont plus atténuées, quoiqu'encore substantielles.
} 
tarification au coût marginal, qui généralise dans un contexte d'externalités la notion de prime équitable en assurance et qu'il sera possible de comparer aux formules de tarification traditionnelles sur base d'assurance individuelle ou d'assurance de responsabilité. L'analyse présentée ici se situe dans le prolongement d'une littérature microéconomique récente portant sur les questions de tarification en assurance automobile. Dans cette littérature, on s'est surtout préoccupé de la relation entre, d'une part, les règles de responsabilité ou le mode de tarification qui pourrait leur être substitué en régime no-fault et, d'autre part, le niveau optimal des activités d'autoprotection des conducteurs. On supposera au contraire ici que les caractéristiques de risque des conducteurs sont exogènes, la seule décision pertinente d'un individu étant celle de conduire ou de ne pas conduire. Plutôt que de considérer des conducteurs homogènes comme on le fait le plus souvent dans cette littérature, on introduira par contre explicitement différentes catégories de risques et de pertes, l'accent étant justement mis ici sur la répartition des coûts entre conducteurs différents ${ }^{5}$.

\section{Tarification et décision de conduire}

Pour simplifier, on supposera que les accidents de la route ne causent des dommages qu'aux utilisateurs de véhicules automobiles (il n'y a pas, par exemple, d'accidents véhiculepiéton) et que ces dommages se résument à des pertes économiques. Soit $R$ le revenu d'un conducteur potentiel et $B$ le bénifice net en équivalent monétaire, sans déduction pour le coût des accidents, qu'il retirera de l'utilisation d'un véhicule. Soit $L$ le dommage qu'il subit en cas d'accident et $p$ sa propablité d'accident ( $L$ et $B$ pourraient dépendre de $R$ ). En l'absence d'assurance et de tout système de responsabilité légale pouvant affecter la répartition des dommages, si $U\left(\right.$.) désigne la fonction d'utilité de l'individu $\left(U^{\prime}>0, U^{\prime \prime} \leq 0\right)$, celui-ci décidera de conduire lorsque

$$
p U(R+B-L)+(1-p) U(R+B)>U(R) .
$$

Avec un système d'assurance obligatoire compensant intégralement la perte $L$, mais requérent le paiement d'une prime $\mathrm{P}$, l'utilité de conduire devient $U(R+B-P)$ et l'individu conduira lorsque

$$
U(R+B-P)>U(R),
$$

c'est-à-dire lorsque $B>P$. Notons que si l'assurance n'était pas obligatoire un individu pourrait préférer conduire sans être assuré, malgré son aversion au risque, lorsque $P$ est suffisamment élevé.

On suppose qu'on peut répartir les conducteurs potentiels en catégories $i=1, \ldots, n$ définies par les caractéristiques de risque des conducteurs et par le dommage qu'ils sont susceptibles de subir en cas d'accident. Soit $X_{i}(P)$ le nombre d'individus de catégorie $i$ pour lesquels le bénifice relié à la possession d'un véhicule est supérieur à la prime $P$ et qui par

\footnotetext{
${ }^{5}$ Sur la base des travaux de Diamond [1974] et Brown [1973], Landes [1982] et Boyer et Dionne [1984], [1985] ont analysé dans un contexte d'externalités directes le rôle de la responsabilité pour négligence comme incitation à la prudence dans la conduite d'une automobile, ainsi que le rôle de la tarification comme substitut à la responsabilité dans les régimes d'assurance no-fault. Ces analyses portent uniquement sur la manière de pratiquer une activité et non sur la décision de la pratiquer ou non (la décision de conduire ici). Shavell [1980a] a montré que la règle de responsabilité pour négligence ne conduit pas à des choix optimaux de niveaux d'activité, bien qu'une responsabilité de plein droit (strict liability) puisse etre appropriée à cette fin. Voir aussi Shavell [1982] pour une analyse de la relation entre le principe de responsabilité et l'assurance de responsabilité.
} 
conséquent décideront de conduire si cette prime est exigée; on a $X_{i}^{\prime}(P)<0$ (sauf pour un $P$ suffisamment grand pour que $X_{i}^{\prime}(P)=X_{i}(P)=0$ ). Avec une prime $P$ obligatoire, le bénéfice total retiré de l'utilisation de véhicules par les individus de catégorie $i$ est :

$$
-\int_{P}^{\infty} B X_{i}^{\prime}(B) d B .^{6}
$$

La probabilité d'accident (par période) d'un conducteur de catégorie $i$ est une fonction $p_{i}\left(X_{1}, \ldots, X_{n}, K\right)$ du nombre de conducteurs de chaque catégorie et d'une variable $K$ représentant la capacité du réseau routier $\left(p_{i j} \equiv \partial p_{i} / \partial X_{j}>0, p_{i K} \equiv \partial p_{i} / \partial K<0\right)$; les fonctions $p_{i}\left(X_{1}, \ldots, X_{n}, K\right)$ sont convexes et homogènes de degré zéro, cette dernière condition définissant implicitement la variable de «capacité». Le coût actuariel total des dommages subis par les conducteurs de catégorie $i$ est:

$$
p_{i}\left(X_{1}, \ldots, X_{n}, K\right) L_{i} X_{i}
$$

Comme il y a un grand nombre de conducteurs, on prend pour acquis que ce coût sera subi avec certitude par le régime d'indemnisation.

Soit $C(K)$ le coût d'un réseau de capacité $K$, où $C^{\prime}>0$. Pour un optimum, la formule de tarification et la capacité devront être choisies de façon à maximiser le bénéfice total net relié à l'utilisation de l'automobile. Celui-ci est égal à la somme des bénéfices individuels, déduction faite du coût des accidents et du coût de la capacité. On résoud ainsi le programme:

$$
\begin{aligned}
\max _{P_{1}, \ldots, P_{n}, K^{i}} & \sum\left\{-\int_{P_{i}}^{\infty} B X_{i}^{\prime}(B) d B\right. \\
& \left.-p_{i}\left[X_{1}\left(P_{1}\right), \ldots, X_{n}\left(P_{n}\right), K\right] L_{i} X_{i}\left(P_{i}\right)\right\}-C(K) .
\end{aligned}
$$

Une solution intérieure à ce problème doit satisfaire:

$$
\begin{aligned}
& P_{i}=L_{i} p_{i}\left(X_{1}, \ldots, X_{n}, K\right)+\sum_{j} L_{j} X_{j} p_{j i}\left(X_{1}, \ldots, X_{n}, K\right), \text { quel que soit } i, \\
& -\sum_{i} p_{i K}\left(X_{1}, \ldots, X_{n}, K\right) L_{i} X_{i}\left(P_{i}\right)=C^{\prime}(K) .
\end{aligned}
$$

La prime optimale $P_{i}$ correspond au coût marginal en accident d'un conducteur potentiel de catégorie $i$. A capacité $K$ donnée, ce coût est égal au coût actuariel $p_{i} L_{i}$ auquel s'expose l'individu en décidant de conduire, augmenté de la variation du coût total en accidents (et donc de la dépense pour le régime d'indemnisation) qu'entraîne sa décision de conduire par suite de l'effet que ceci aura sur la probabilité d'accident de tous les conducteurs. Un individu n'accédera ainsi au réseau routier que si le bénéfice $B$ qu'il en retire est supérieur au coût $P_{i}$ qu'il impose à la collectivité. Notons qu'on aurait pu obtenir (4a) et (4b) à partir d'une fonction de préférences sociales quelconque en optimisant simultanément par rapport à la répartition des revenus et par rapport à l'utilisation de l'automobile.

${ }^{6}$ Cette expression est égale à l'expression plus familière

$$
\int_{0}^{X_{i}(P)} B_{i}(X) d X
$$

où $B_{i}(X)$ est la fonction inverse de $X_{i}(P)$. 


\section{Choix de capacité}

Le terme d'effets externes apparaissant dans le membre de droite de l'équation (4a) est en partie un effet de congestion pur égal à la variation marginale du coût total en accidents attribuable à une augmentation proportionnelle de la population des différentes catégories d'automobilistes. La capacité du réseau étant une variable de décision, c'est cet effet qui sera pris en compte dans le choix de capacité. Soient $X=\Sigma X_{i}$ la population totale de conducteurs et $x_{i}=X_{i} / X$ la proportion de conducteurs de catégorie $i$. Soient:

$$
\begin{aligned}
\hat{p}_{j i} & \equiv p_{j i}-\sum_{k} x_{k} p_{j k}, \\
p_{j X} & \equiv \frac{\partial}{\partial X} p_{j}\left(x_{1} X, \ldots, x_{n} X, K\right) \\
& =\sum_{k} p_{j k}\left(X_{1}, \ldots, x_{n}, K\right) x_{k} .
\end{aligned}
$$

En utilisant l'identité $p_{j i}=p_{j X}+\hat{p}_{j i}$, le terme d'externalités peut s'écrire sous la forme:

$$
\sum_{j} p_{j i} L_{j} X_{j}=\sum_{j} p_{j X} L_{j} X_{j}+\sum_{j} \hat{p}_{j i} L_{j} X_{j}
$$

Le premier terme du membre de droite de (7) est indépendant de $i$ et correspond à l'effet de congestion pur découlant d'une augmentation proportionnelle de la population de conducteurs. Si les caractéristiques de risque des conducteurs étaient telles que les externalités générées par chaque catégorie d'automobilistes étaient identiques $\left(p_{i i}=p_{j k}\right.$ pour $\left.i \neq k\right)$, le second terme serait nul. Même lorsque ceci n'est pas le cas, on vérifie cependant que ce terme est nul en moyenne:

$$
\begin{gathered}
\sum_{\mathrm{i}} x_{i}\left(\sum_{\mathrm{j}} \hat{p}_{j i} L_{j} X_{j}\right)=\sum_{\mathrm{j}} L_{j} X_{j}\left(\sum_{\mathrm{i}} p_{j i} x_{i}-p_{j X}\right) \\
=0 .
\end{gathered}
$$

Il s'ensuit que les primes perçues au total satisfont:

$$
\sum_{i} X_{i} P_{i}=\sum_{i} p_{i} L_{i} X_{i}+X \sum_{i} p_{i X} L_{i} X_{i}
$$

où $\Sigma p_{i} L_{i} X_{i}$ est le coût total en accidents (c'est donc ce que devra percevoir le régime d'assurance pour équilibrer son budget) et où $X \Sigma p_{i X} L_{i} X_{i}$ est l'effet de congestion total. Si la capacité du réseau était fixe, ce dernier terme serait assimilable à la somme des taxes de congestion qu'il faudrait percevoir auprès des automobilistes dans le but de rationner leur accès au réseau. Lorsque la capacité est variable, ce qu'on suppose ici, le terme en question sera égal à la part des automobilistes dans le financement de la capacité du réseau.

Les fonctions $p_{i}$ étant homogènes de degré zéro, on a:

$$
\sum_{j} p_{i j} X_{j}+p_{i K} K=0 \text {. }
$$

A partir de (4b), on peut par conséquent écrire:

$$
\begin{aligned}
K C^{\prime}(K) & =-K \sum_{i} p_{i K} L_{i} X_{i} \\
& =\sum_{i}\left(\sum_{j} p_{i j} X_{j}\right) L_{i} X_{i} \\
& =X \sum_{i}\left(\sum_{j} p_{i j} x_{j}\right) L_{i} X_{i} \\
& =X \sum_{i} p_{i X} L_{i} X_{i} .
\end{aligned}
$$

Si l'offre de capacité est parfaitement élastique, c'est-à-dire $C$ "' $(K)=0$ pour tout $K$, on aura évidemment $K C^{\prime}(K)=C(K)$, de sorte que le terme de congestion agrégé sera tout simplement égal au coût total de la capacité. De façon générale, on aura $K C^{\prime}(K) \lesseqgtr C(K)$ selon 
qu'il y a des rendements croissants ou décroissants dans l'obtention de capacité. Selon le cas, on considérera alors que l'organisme chargé du réseau routier opère à perte, ses pertes étant couvertes par une subvention de l'Etat financée par l'impôt, ou qu'au contraire il perçoit un surplus, au profit de l'Etat, par le biais de taxes de congestion pures. Dans ce dernier cas, c'est-à-dire lorsque $K C^{\prime}(K)>C(K)$, on peut assimiler $C^{\prime}(K)$ au prix de marché $q$ que doivent payer les utilisateurs pour l'obtention d'une unité de capacité. L'effet de congestion agrégé est alors égal à la dépense totale $C^{\prime}(K) K \equiv q K$ que représente une capacité $K$, la dépense en question incluant la rente de certains facteurs de production, par exemple la rente foncière (c'est alors cette rente qui joue le rôle d'une taxe de congestion pure).

La proposition qui suit résume les implications des resultats précédents pour la formule de tarification:

Proposition 1. Le péage optimal $P_{i}$ a la forme

$$
\begin{array}{ll}
P_{i}=T+\Pi_{i}, & \text { où } \\
T \equiv C^{\prime}(K) K / X, &
\end{array}
$$

$$
\Pi_{i} \equiv p_{i} L_{i}+\sum_{j} \hat{p}_{j i} X_{j} L_{j}
$$

$T$ est la participation uniforme de chaque conducteur au financement de la capacité du réseau et $\Pi_{i}$ sa participation personnalisée au financement de l'indemnisation des accidents de la route, les $\Pi_{i}$ satisfaisant

$$
\sum_{\mathrm{i}} \Pi_{i} X_{i}=\sum_{i} p_{i} L_{i} X_{i} \|
$$

Les $\Pi_{i}$ s'interprètent comme des coûts marginaux d'accidents en l'absence d'effet de congestion, c'est-à-dire à coefficient de capacité $\kappa \equiv K / X$ donné:

$$
\Pi_{i}=\frac{\partial}{\partial X_{i}} \sum_{j} p_{j}\left(X_{1}, \ldots, X_{n}, \kappa X\right) L_{j} X_{j},
$$

où l'on obtient (15) en notant que $X$ varie avec $X_{i}$. On pourrait considérer que $T$ et $\Pi_{i}$ sont perçus par deux organismes distincts, l'un s'occupant du choix de capacité, l'autre de l'indemnisation des victimes d'accidents. A l'équilibre, on aurait ainsi la situation suivante:

(i) L'organisme chargé du réseau routier considère les $X_{i}$ comme donnés et choisit $K$ de façon à minimiser la somme du coût en accidents et du coût de capacité. Il finance le coût $C(K)$ en percevant auprès de chaque conducteur un péage uniforme $T$ (une vignette annuelle par exemple) relié au coût marginal de la capacité, quitte le cas échéant à reverser ses excédents à l'Etat ou à recevoir de celui-ci une subvention pour couvrir ses pertes. L'activité de cet organisme détermine le ratio $K / X$.

(ii) L'organisme d'assurance condidère comme donné le coefficient de capacité $K / X$. Il finance les indemnités versées $\Sigma p_{i} L_{i} X_{i}$ en percevant des primes d'assurance $\Pi_{i}$ égales au coût marginal que représente chaque automobiliste à coefficient de capacité donné, en négligeant l'effet de congestion. Cet organisme s'autofinance, sans surplus ni perte?

${ }^{7}$ La proposition démontre la possibilité de séparer d'un point de vue organisationnel le financement de la «capacité» du réseau routier et celui de l'assurance obligatoire, même s'il y a en fait une relation fonctionnelle entre la capacité du réseau et le coût total en accidents. Mais rien n'empêche évidemment qu'à l'instar de ce qui se fait dans plusieurs pays (voir par exemple les travaux de la CNU. CED [1987] sur ce point) les mêmes assiettes soient utilisées pour financer les deux activités: droit sur le permis de conduire, vignette annuelle, taxes sur l'essence, etc. Le financement de l'assurance automobile en partie sur la base de taxes sur l'essence, comme dans la proposition de Hoffer et Miller [1979], a été pratiqué au Québec de 1978 à 1980. 
L'emploi d'une taxe uniforme pour rationner la consommation d'un bien collectif sujet à congestion est bien connu. On utilise cependant ici l'idée que ce péage sera relié au financement d'une capacité optimale du bien en question lorsque la capacité est adaptable (cf. Oakland,[1972]). Ceci facilite la comparaison entre une formule de tarification marginale de l'assurance (qui devrait inclure une taxe de congestion si la capacité était fixe) et des formules non optimales qui sont des variantes d'une tarification au coût moyen et qui, par conséquent, ne prennent pas en compte l'externalité de congestion. En effet, nous discuterons plus loin de diverses formules de tarification de l'assurance (primes uniformes, primes sur base d'assurance individuelle ou sur base de responsabilité). Chacune de ces formules répartit différemment le coût total de l'assurance (qui peut aussi être différent d'une formule à l'autre); elles sont donc en ce sens des tarifications au coût moyen, quoique non nécessairement uniformes. De ce point de vue, la prime d'assurance au coût marginal $\Pi_{i}$ correspond aussi à une répartition du coût total de l'assurance. Quelle que soit la formule de tarification retenue en assurance, il est raisonnable de supposer que dans chaque cas les pouvoirs publics choisissent $K$ indépendamment de façon à minimiser le coût des accidents, compte tenu du coût de capacité, et qu'ils facturent à chaque automobiliste un péage $T$ en conséquence. Ce péage ayant pour effet d'internaliser le coût de congestion lié à l'utilisation du réseau routier, ceci nous permettra de comparer les tarifications utilisées pour l'assurance proprement dite, en faisant abstraction, pour l'essentiel, du péage $T$ de congestion ou de financement de la capacité.

\section{Facteurs de risque individuels et externalités}

Pour discuter des différente formules possibles de tarification de l'assurance, il sera intéressant dans ce qui suit de décrire plus explicitement les interactions entre automobilistes et de le faire en identifiant, dans un cadre causal, les facteurs de risque propres à chaque catégorie de conducteurs. On supposera pour simplifier que tous les accidents impliquent deux véhicules. Un accident ne pouvant ainsi se produire que lors d'une «rencontre» entre deux véhicules, les probalités d'accident par période seront fonction de la fréquence de rencontres (ou fréquence des «occasions d'accident») et de la probabilité qu'un accident se produise à l'occasion d'une rencontre. Soit $q_{i j}$ la probabilité conditionnelle qu'un accident se produise à la suite d'une rencontre entre des automobilistes de catégories $i$ et $j$. On fait l'hypothèse que $q_{i j}$ est additive dans les facteurs de risque des conducteurs impliqués, c'est-àdire

$$
q_{i j}=q_{i}+q_{j}
$$

où les $q_{i}, q_{j}$ sont constants. On interprétera $q_{i}$ comme la probabilité pour un conducteur $i$ de "provoquer» un accident lors d'une rencontre (par suite d'une maladresse ou d'une négligence de sa part, celles-ci pouvant être dues aussi bien à sa façon de conduire qu'à un événement fortuit quelconque $)^{8}$.

${ }^{8}$ L'hypothèse selon laquelle un accident implique nécessairement deux véhicules est standard. Voir par exemple Diamond [1974], Landes [1982] et Boyer et Dionne [1984], [1985b]. Ces auteurs analysent les configurations optimales de comportement d'autoprotection des agents, alors que ces comportements sont considérés comme donnés ici. Dans la perspective de ces auteurs, la probabilité d'un accident $i j$ serait proportionnelle à la fonction $q\left(y_{i}, y_{j}\right)$ où $y_{i}, y_{j}$ sont les variables de comportement. L'hypothèse d'addivité de cette fonction, $q_{i j}\left(y_{i}, y_{j}\right)=\mathrm{q}_{\mathrm{i}}\left(y_{i}\right)+\mathrm{q}_{\mathrm{j}}\left(y_{j}\right)$, a été utilisée en particulier par Green [1976] et Shavell [1985]. On suppose alors que les événements « $i$ cause un accident» et « $j$ cause un accident» sont disjoints. Si on admet qu'un accident puisse être causé indépendamment par les deux conducteurs à la fois, on aurait $q_{i j}=q_{i}+q_{j}-q_{i} q_{p}$, ce qui ne modifierait pas sensiblement l'analyse. 
Si tous les conducteurs ont le même degré d'exposition au risque (kilomètres parcourus ou fréquence d'utilisation du véhicule par période, etc.), la probabilité qu'une rencontre entre un conducteur $i$ et un autre conducteur se fasse avec un conducteur de catégorie $j$ est donnée par $x_{j}$. Pour un conducteur $i$, la probabilité d'accident par rencontre, $\bar{q}_{i}$, est donc:

$$
\begin{aligned}
\bar{q}_{i} & =\sum_{j} q_{i} x_{j} \\
& =q_{i}+\bar{q},
\end{aligned}
$$

où $\bar{q}=\Sigma x_{j} q_{j}$ est le facteur de risque moyen. Enfin, la fréquence de rencontre par automobiliste est une fonction $h(X, K)$ du nombre de véhicules en circulation et de la capacité du réseau $\left(h(X, K)\right.$ est convexe et homogène de degré zéro, avec $h_{X}>0$ et $\left.h_{K}<0\right)$. La probabilité d'accident d'un conducteur $i$ est alors:

$$
p_{i}=h(X, K) \bar{q}_{i} \text {, }
$$

ce qui correspond à une spécification particulière de la fonction $p_{i}\left(X, \ldots, X_{n}, K\right)$. Notons que l'hypothèse selon laquelle la fréquence de rencontres avec un automobiliste de type $j$ est proportionnelle à la part de ces automobilistes dans le total de conducteurs pourrait être levée sans difficulté. On pourrait ainsi supposer que les différentes catégories d'automobilistes sont caractérisées par des degrés d'exposition au risque différents, sur la base du nombre de kilomètres parcourus ou de la fréquence d'utilisation de l'automobile. Ces modifications ne changeraient rien d'essentiel.

Avec cette spécification, on obtient:

$$
\begin{aligned}
p_{j i}\left(X_{1}, \ldots, X_{n}, K\right) & =\mathrm{h}_{\mathbf{X}}(X, K) \bar{q}_{j}+h(X, K)\left(q_{i}-\bar{q}\right) / X \\
& =[h(X, K) / X]\left[\varepsilon(X, K) \bar{q}_{j}+q_{i}-\bar{q}\right]
\end{aligned}
$$

où

$$
\varepsilon(X, K)=X h_{X}(X, K) / h(X, K)
$$

est l'élasticité de la fréquence de rencontres par rapport au nombre de véhicules. La fonction $h$ étant convexe (d'où $h_{X X} \geq 0$ ) et en supposant $h(0, K)=0$, on a $\varepsilon \geq 1$, ce qui garantit $p_{j i}>0$. A partir de (17), (18) et (19), le péage optimal $P_{i}$ de l'équation (4a) peut se réécrire:

$$
\begin{aligned}
P_{i} & =p_{i} L_{i}+\sum(h / X)\left(\varepsilon \bar{q}_{j}+q_{i}-\bar{q}\right) L_{j} X_{j} \\
& =p_{i} L_{i}+\varepsilon \sum_{j} h \bar{q}_{j} L_{j} x_{j}+h\left(q_{i}-\bar{q}\right) \bar{L} \\
& =p_{i} L_{i}+\varepsilon \sum_{j} h \bar{q}_{j} L_{j} x_{j}+\left[h\left(q_{i}+\bar{q}\right)-2 h \bar{q}\right] \bar{L} \\
& =p_{i} L_{i}+\varepsilon \sum_{j} p_{j} L_{j} x_{j}+\left(p_{i}-\bar{p}\right) \bar{L},
\end{aligned}
$$

où $L=\Sigma L_{f} x_{j}$ est la perte moyenne et où $\bar{p}$ est la probabilité moyenne d'accidents:

$$
\begin{aligned}
\bar{p} & =\sum_{j} p_{f} x_{j} \\
& =2 \bar{q} h(X, K),
\end{aligned}
$$

En utilisant (11) et (13a), on peut vérifier par ailleurs que la condition (4b) caractérisant la capacité optimale devient ici:

$$
\varepsilon \sum_{j} p_{j} L_{r} x_{j}=K C^{\prime}(K) / X \equiv T .
$$

En écrivant, comme dans la section précédente, le péage optimal $P_{i}$ comme la somme d'une taxe $T$ de congestion ou de financement de la capacité et d'une prime d'assurance 
proprement dite $\Pi_{i}$, on obtient par conséquent pour cette dernière:

$$
\Pi_{i}=p_{i} L_{i}+\left(p_{i}-\bar{p}\right) \bar{L} \text {. }
$$

Afin de comparer cette prime aux formules plus traditionnelles de tarification de l'assurance, nous utiliserons aussi l'expression équivalente (qui se déduit de (17), (18) et (22)):

$$
\Pi_{i}=h\left(q_{i}+\bar{q}\right) L_{i}+h\left(q_{i}-\bar{q}\right) \bar{L} .
$$

Il peut être intéressant de noter que lorsque tous les conducteurs sont identiques, c'està-dire $L_{i}=\bar{L}$ et $q_{i}=\bar{q}$ pour tout $i$ (et donc $p_{i}=\bar{p}$ ), et lorsque $\varepsilon=1$, le péage total optimal $P_{i}$ de l'équation (21) devient $P_{i}=2 \bar{p} \bar{L}$. Ceci rejoint un résultat obtenu par Diamond [1974], Landes [1982] et Boyer et Dionne [1985], ce résultat apparaissant donc comme un cas particulier de l'analyse présentée ici. Ces auteurs faisaient cependant implicitement l'hypothèse que la «capacité» était constante et ils interprétaient $P_{i}$ comme la prime d'assurance optimale. Selon l'approche retenue ici, la prime d'assurance proprement dite $\Pi_{i}$ est égale à la valeur actuarielle du dommage $\bar{p} \bar{L}$ quand les conducteurs sont homogènes, la taxe de congestion ou de financement de la capacité $T$ étant dans ce cas elle-même aussi égale à $\bar{p} \bar{L}$. Enfin on peut faire remarquer que le cas $\varepsilon=1$ correspond à une situation où le nombre total de «rencontres» que peut faire un automobiliste (à capacité donné du réseau) est proportionnel au nombre de véhicules en circulation.

\section{Formules de tarification}

Trois formules de tarification de l'assurance ont déjà été introduites de façon plus ou moins explicite. Soient $\Pi^{u}, \Pi_{i}^{d}$ et $\Pi_{i}^{m}$ les tarifications uniformes, sur base d'assurance individuelle (assurance directe) et au coût marginal respectivement. $\Pi_{i}^{m}$ est défini par les équations (24a) ou (24b), $\Pi^{u}$ et $\Pi_{i}^{d}$ sont données par:

$$
\begin{aligned}
& \Pi^{u}=\sum_{j} p_{j} L_{j} x_{j}=h \sum L_{j}\left(q_{j}+\bar{q}\right) x_{j}, \\
& \Pi_{i}^{d}=p_{i} L_{i}=h\left(q_{i}+\bar{q}\right) L_{i} .
\end{aligned}
$$

Pour chacune de ces formules, le péage total imposé au conducteur $i$ est de la forme:

$$
P_{i}^{s}=T^{s}+\Pi_{i}^{s} \quad(s=u, r, m),
$$

où $T^{s}$ est le coût par conducteur, $C^{\prime}(K) K / X$, du financement de la capacité, celle-ci étant choisie dans chaque cas de façon à résoudre:

$$
\min _{\mathbf{K}}\left\{\sum_{\mathrm{j}} p_{j} L_{j} X_{j}+C(K)\right\} \text {. }
$$

A chaque formule de tarification est ainsi associé un équilibre défini par les foncitions de demande $X_{i}\left(P_{i}^{s}\right)$, les formules de tarification elles-mêmes en (27) et la condition optimale (23). Chacun de ces équilibres pouvant être caractérisé par une population différente de conducteurs, notamment en ce qui concerne la composition du bassin d'automobilistes, les $T^{s}$ pourront différer selon la tarification choisie par l'assurance.

Qu'en serait-il maintenant d'une tarification sur base de responsabilité? Dans un régime d'assurance obligatoire compensant intégralement les dommages de toutes les victimes, une tarification sur base de responsabilité de plein droit consisterait à faire payer à un conducteur le coût actuariel des dommages qu'il est susceptible de causer. La probalité qu'à l'occasion d'une rencontre $i j$ un accident se produise par suite du fait du conducteur $i$ est $q_{i}$ La probabilité qu'une telle rencontre se produise pour ce conducteur est $h x_{j}$ et le coût de l'accident est $L_{i}+L_{i}$. Le coût des accidents imputables statistiquement au conducteur $i$ dans 
un régime de responsabilité de plein droit est donc $h q_{i}\left(L_{i}+\bar{L}\right)$. Dans un régime de responsabilité pour négligence, un conducteur ne sera tenu responsable des dommages infligés aux tiers que s'il est reconnu négligent. Soit $\alpha_{i}$ la probalité conditionnelle que le conducteur $i$ soit reconnu responsable des suites d'un accident de son fait; $\alpha_{i}$ pourra dépendre du critère de négligence utilisé et du comportement de l'automobiliste. Le coût actuariel de sa responsabilité à l'égard des tiers est alors $h q_{i} \alpha_{i} L$. Comme il y a compensation intégrale de tous les dommages, ce conducteur devra aussi assumer, sur une base d'assurance individuelle, le coût de ses propres dommages, lorsque ceux-ci sont attribuables à ses propres actions ou au fait non fautif des autres conducteurs. Le coût de cette protection est:

$$
L_{i} h q_{i}+L_{i} \sum h q_{j}\left(1-\alpha_{j}\right) x_{j} .
$$

La prime d'assurance totale dans le cas d'une tarification sur base de responsabilité est donc:

où

$$
\begin{aligned}
\Pi_{i}^{r} & =\bar{L} h q_{i} \alpha_{i}+L_{i} h q_{i}+L_{i} \sum h q_{j}\left(1-\alpha_{j}\right) x_{j} \\
& =h q_{i}\left(L_{i}+\bar{L}\right)+\left[L_{i} \sum_{j} h q_{j}\left(1-\alpha_{j}\right) x_{j}-\bar{L} h q_{i}\left(1-\alpha_{i}\right)\right] \\
& =h q_{i}\left(L_{i}+\bar{L}\right)+h\left(\bar{\beta} L_{i}-\beta_{i} \bar{L}\right),
\end{aligned}
$$

$$
\beta_{i} \equiv q_{i}\left(1-\alpha_{i}\right)
$$

est la probabilité qu'un automobiliste $i$, à l'occasion d'une rencontre, cause un accident sans être tenu responsable du dommage aux tiers et où $\bar{\beta} \equiv \Sigma \beta_{j} x_{j}$ est la probabilité moyenne de provoquer un accident sans imputation de responsabilité . On a évidemment $0 \leq \beta_{i} \leq q_{i}$. Lorsque $\beta_{i}=0$, on retrouve la responsabilité stricte; lorsque $\beta_{i}=q_{i}, \Pi_{i}^{r}$ est égal à la prime d'assurance individuelle $\Pi_{i}^{d}$. La formulation (30) est compatible aussi avec la responsabilité objective (sans faute) avec exonération pour cas fortuit. Il est raisonnable de supposer que la probabilité (par «rencontre») d'une situation de cas fortuit est la même pour tous. On aura alors $\beta_{i}=\beta$ où $\beta<q_{i}$ pour tout $i$, la constante $\beta$ étant interprétée comme la probabilité qu'un conducteur cause un accident par suite de circonstances jugées inévitables (et justifiant par conséquent une exonération de responsabilité) ${ }^{9}$.

Par rapport à la tarification au coût marginal $\Pi_{i}^{m}$, les tarifications $\Pi_{i}^{d}$ et $\Pi_{i}^{r}$ font toutes deux apparaître une forme d'externalités. En faisant abstraction des effets de structure (c'est-à-dire pour des $x_{j}$ identiques), on peut écrire en effet, en comparant (24b) et (26),

$$
\Pi_{i}^{m}=\Pi_{i}^{d}+h \bar{L}\left(q_{i}-\bar{q}\right)
$$

où l'on peut vérifier que:

$$
h \bar{L}\left(q_{i}-\bar{q}\right)=\sum_{j} X_{j}\left(\partial \Pi_{j}^{d} / \partial X_{i}\right) \mid \kappa \text { constant } .
$$

La prime d'assurance optimale peut ainsi s'exprimer comme la somme de la prime d'assurance individuelle no-fault et d'un facteur correspondant à la hausse des primes d'assurance individuelle provoquée par la présence de l'agent considérée, abstraction faite de l'effet de congestion (c'est-à-dire à coefficient de capacité $\kappa$ donné).

\footnotetext{
${ }^{9}$ Pour une description des différentes règles de responsabilité utilisées en assurance automobile selon les pays, voir Tunc [1981a] et [1981b]. Pour une analyse formalisée des règles de responsabilité, notamment en ce qui concerne l'équivalent du cas fortuit, voir Shavell [1980b]. On pourrait considérer explicitement les comportements des automobilistes, en écrivant par exemple $q_{i}\left(y_{i}\right)$ où $y_{i}$ est la variable de comportement. Il est implicite dans la formulation retenue ici qu'un individu ne contrôle que de façon stochastique sa probabilité de commettre une «négligence» engageant la responsabilité (cf. Diamond, [1974]).
} 
De même, pour les primes d'assurances sur base de responsabilité, on a à partir de (24b) et (30):

$$
\Pi_{i}^{m}=\Pi_{i}^{r}+h \bar{q}\left(L_{i}-\bar{L}\right)+h\left(\beta_{i} \bar{L}-\bar{\beta} L_{i}\right),
$$

où

$$
h \bar{q}\left(L_{i}-\bar{L}\right)+h\left(\beta_{i} \bar{L}-\bar{\beta} L_{i}\right)=\left.\Sigma X_{j}\left(o \Pi_{j}^{r} / \delta X_{i}\right)\right|_{\kappa} \text { constant }
$$

La prime d'assurance optimale est ici égale à la prime sur base de responsabilité corrigée d'un facteur correspondant à la hausse des primes sur base de responsabilité infligée par la présence de l'agent. Bien que $\Pi_{i}^{d}$ et $\Pi_{i}^{r}$ ne soient pas optimales en général, il est clair qu'elles seront équivalentes à $\Pi_{i}^{m}$ dans certaines circonstances, par exemple lorsque dans (31) $q_{i}$ $=\bar{q}$ pour tout $i$ ou lorsque dans (33) $L_{i}=\bar{L}$ et $\beta_{i}=\bar{\beta}$ pour tout $i$. Dans ces circonstances, les taxes de congestion $T^{d}$ et $T^{r}$ seront aussi égales $T^{m}$. La comparaison entre les différentes tarifications est résumée dans la proposition qui suit:

Proposition 2. De façon générale, aucune des formules de tarification traditionnelles n'est optimale. Cependant,

(i) lorsque les facteurs de risque et les dommages sont les mêmes chez tous les conducteurs, toutes les formules de tarification sont équivalentes (prime uniforme, sur base d'assurance individuelle, sur base de responsabilité stricte ou pour négligence et prime au cô̂t marginal);

(ii) lorsque les facteurs de risque sont identiques, la tarification sur base d'assurance individuelle est optimale;

(iii) lorsque les dommages sont identiques, la tarification sur base de responsabilité stricte, avec ou sans exonération pour le cas fortuit (c'est-à-dire $\beta_{i}=0$ ou $\beta_{i}=\beta$ pour tout $i$ ), est optimale. La tarification sur base de responsabilité pour négligence (avec en général $\beta_{i} \neq \beta_{j}$, pour $\left.i \neq j\right)$ n'est cependant pas optimale.| I

\section{Interfinancement}

On émet souvent l'opinion que dans un système no-fault (avec primes d'assurance individuelle ou a fortiori avec des primes uniformes) les «bons» conducteurs subventionnent les «mauvais» par opposition à ce qu'on observerait avec le principe de responsabilité civile. Le critère adopté ici pour juger de l'existence d'interfinancement est l'écart par rapport à une tarification au coût marginal. Sur cette base, en se référant aux équations (31) et (33), s'il est vrai qu'avec l'assurance individuelle les conducteurs à facteurs de risque élevés sont avantagés par rapport à une tarification marginale, il serait tout aussi juste de dire que dans un système de responsabilité stricte $\left(\beta_{i}=\beta\right.$ pour tout $\left.i\right)$ les conducteurs à dommage élevé sont également favorisés relativement à la prime au coût marginal. Ces équations ne permettent pas cependant de comparaison directe entre les primes d'assurance individuelle et sur base de responsabilité. Pour un conducteur à perte $L_{i}$ et à facteur de risque $q_{i}$ donnés, il est évidemment possible de calculer, de manière hypothétique (c'est-à-dire pour des $X_{i}$ donnés), laquelle des formules de tarification il préférerait. L'opinion ci-dessus sur les effets redistributifs entre «bons» et «mauvais» conducteurs porte toutefois sur une comparaison en moyenne entre classes de risque. Il est clair que pour faire une telle comparaison de façon véritablement satisfaisante il faudrait tenir compte du fait que chaque tarification détermine un ensemble différent de conducteurs sur la route, ce qui affectera $h, \bar{q}$ et $\bar{L}$. On raisonnera néammoins ici à populations $X_{i}$ données,ce qui suffit pour obtenir une indication 
du sens des avantages ou désavantages relatifs entre catégories de risque ou catégories de dommage selon la formule de tarification.

A partir des $x_{i}$, on obtient une fonction de probabilité conjointe sur les dommages et les facteurs de risque $L$ et $q$ considérés comme variables aléatoires. En normalisant pour que $h(X, K)=1$, la prime uniforme peut s'écrire:

$$
\begin{aligned}
\Pi^{u} & =E[(q+\bar{q}) L] \\
& =2 \bar{q} \bar{L}+S_{L q},
\end{aligned}
$$

où $E$ l'opérateur d'espérance mathématique et où $S_{L q}$ est la covariance entre $L$ et $q$. Les primes payées en moyenne pour des conducteurs d'une catégorie de risque $q$ donnée s'écrivent alors:

$$
\bar{\Pi}^{s}(q)=E\left[\Pi^{s}(L, q) \mid q\right] \quad(s=d, r, m) .
$$

De même, pour une catégorie de dommage $L$ donnée, la prime payée en moyenne est:

$$
\bar{\Pi}^{s}(L)=E\left[\Pi^{s}(L, q) \mid L\right] \quad(s=d, r, m) .
$$

Soient $\bar{L}_{q}=E(L \mid q)$ et $\bar{q}_{L}=E(q \mid L)$ les moyennes conditionnelles. Avec cette notation,et en ne retenant que le concept de responsabilité stricte pour le cas des primes sur base de responsabilité, les primes payées en moyenne par différentes catégories de risque ou de dommage satisfont:

$$
\begin{aligned}
& \bar{\Pi}^{m}(q)=(q+\bar{q}) \bar{L}_{q}+(q-\bar{q}) \bar{L}, \\
& \bar{\Pi}^{d}(q)=(q+\bar{q}) \bar{L}_{q}, \\
& \bar{\Pi}^{r}(q)=q\left(\bar{L}_{q}+\bar{L}\right), \\
& \bar{\Pi}^{m}(L)=\left(\bar{q}_{L}+\bar{q}\right) L+\left(\bar{q}_{L}-\bar{q}\right) \bar{L}, \\
& \bar{\Pi}^{d}(L)=\left(\bar{q}_{L}+\bar{q}\right) L, \\
& \bar{\Pi}^{r}(L)=\bar{q}_{L}(L+\bar{L}) .
\end{aligned}
$$

Lorsque les facteurs de risque et les dommages sont statistiquement indépendants au sein de la population de conducteurs, on a $S_{L q}=0$, ainsi que $\bar{L}_{q}=\bar{L}$ et $\bar{q}_{L}=\bar{q}$. On obtient alors:

$$
\begin{aligned}
& q>\bar{q} \bar{\Pi}^{m}(q)=\bar{\Pi}^{r}(q)>\bar{\Pi}^{d}(q)>\Pi^{u}, \\
& L>\bar{L} \bar{\Pi}^{m}(L)=\bar{\Pi}^{d}(L)>\bar{\Pi}^{r}(L)>\Pi^{u},
\end{aligned}
$$

les inégalités étant inversées pour $q<\bar{q}$ ou $L<\bar{L}$.

Ces inégalités s'interprètent de la façon suivante: par exemple,le fait que $\bar{\Pi}^{r}(q)>$ $\bar{\Pi}^{d}(q)$ pour $q>\bar{q}$ signifie que les conducteurs à facteur de risque «élevé» (ceux pour lesquels $q>\bar{q}$ ) préféreront en moyenne une tarification sur base d'assurance individuelle à une tarification sur base de responsabilité; le fait que $\bar{\Pi}^{m}(q)>\bar{\Pi}^{d}(q)$ pour $q>\bar{q}$, ou de façon équivalente que $\bar{\Pi}^{m}(q)<\bar{\Pi}^{d}(q)$ pour $q<\bar{q}$, signifie qu'avec une tarification sur base d'assurance individuelle les conducteurs à facteur de risque «modéré» (pour lesquels $q<\bar{q}$ ) subventionnent en moyenne les conducteurs à facteur de risque élevé, le critère pour décider s'il y a interfinancement étant ici la comparaison ave la tarification au coût marginal. L'interprétation est la même, pari passu, lorsqu'on compare les formules de tarification pour les conducteurs d'une catégorie de dommages donnée: $\bar{\Pi}^{d}(L)>\Pi^{r}(L)$ pour $L>\bar{L}$ signifie 
les conducteurs à dommage élevé $(L>\bar{L})$ préfèreront en moyenne l'assurance sur base de responsabilité à l'assurance individuelle ; $\bar{\Pi}^{m}(L)>\bar{\Pi}^{r}(L)$ pour $L>L$ signifie qu'avec une tarification sur base de responsabilité les conducteurs à dommage modéré $(L<\bar{L})$ subventionnent en moyenne les conducteurs à dommage élevé.

Lorsque les caractéristiques ne sont pas indépendantes, le classement des formules de tarification sera modifié en partie. En l'absence d'information sur la nature exacte de la fonction de probabilité conjointe reliant $L$ et $q$, on peut admettre qu'avec une corrélation positive $\left(S_{L q}>0\right)$, on aura $\bar{L}_{q}>\bar{L}$ lorsque $q>>\bar{q}(q$ «suffisamment» supérieur à $\bar{q})$ et $\bar{q}_{L}$ $>\bar{q}$ lorsque $L>>\bar{L}$; avec une corrélation négative $\left(S_{L q}<0\right), q>>\bar{q}$ implique $\bar{L}_{q}<\bar{L}$ et $L>>\bar{L}$ implique $\bar{q}_{L}<\bar{q}$. A partir d'un développement analogue à celui qui a été utilisé pour le cas où les caractéristiques étaient indépendantes, on obtient ainsi le classement représenté au tableau 1.

On remarque que lorsque $S_{q L}<0$ la prime uniforme ne peut être classée par rapport à aucune autre formule de tarification sans information additionnelle, bien qu'on puisse classer entre elles $\bar{\Pi}^{d}, \bar{\Pi}^{r}$ et $\bar{\Pi}^{m}$. Ceci tient au fait que chacune de ces tarifications est personnalisée en ce sens qu'elle tient compte de $q$ et de $L$; avec une corrélation négative, ces deux caractéristiques tendent à se compenser par rapport à une tarification uniforme. Pour des raisons analogues, il n'est pas possible sans information additionnelle de classer entre elles $\bar{\Pi}^{d}$ et $\bar{\Pi}^{r}$ lorsque $S_{q L}>0$. On peut vérifier cependant qu'avec les hypothèses faites ici $q>>$ $\bar{q}$ implique toujours $\bar{\Pi}^{m}(q)>\bar{\Pi}^{d}(q)$, quel que soit le signe de $S_{L q}$; de même, $L>>\bar{L}$ implique toujours $\bar{\Pi}^{m}(L)>\bar{\Pi}^{r}(L)$. En d'autres termes, avec une tarification sur base d'assurance individuelle les «bons» facteurs de risque subventionnent toujours les «mauvais» en moyenne, alors qu'avec une tarification sur base de responsabilité les conducteurs à dommage modéré subentionnent toujours en moyenne les conducteurs à dommage élevé. La proposition 3 résume l'essentiel des résultats obtenus ${ }^{10}$.

${ }^{10}$ Lorsque $S_{L q} \neq 0$, le classement de $\bar{\Pi}^{d}, \Pi^{r}$ et $\bar{\Pi}^{m}$ apparaissant au tableau 1 se dérive directement des équations (38a) à (39c) si on suppose que $L \gg \bar{L}$ et $q>\bar{q}$ impliquent respectivement $\bar{q}_{L}>\bar{q}$ et $\bar{L}_{q}>\bar{L}$. Lorsque $S_{L q}>0$, le classement de $\Pi^{u}$ par rapport aux autres formules de tarification peut se démontrer en faisant l'hypothèse additionnelle d'une relation linéaire entre $\bar{q}_{L}$ et $L$ ou entre $\bar{L}_{q}$ et $q$ (ce sera le cas si $q$ et $L$ satisfont une loi normale bivariée). A titre d'exemple on démontrera que $\bar{\Pi}^{r}(q)>$ $\Pi^{u}$ si $q>\bar{q}$, En réécrivant (35) sous la forme:

$\Pi^{u}=E(q L)+\bar{q} \bar{L}$,

on a, pour $q$ donné, compte tenu de (38c),

$\bar{\Pi}^{r}(q)-\Pi^{u}=(q-\bar{q}) \bar{L}+q \bar{L}_{q}-E(q L)$.

Avec une relation linéaire, on a:

$\bar{L}_{q}=\bar{L}+\gamma(q-\bar{q})$,

où $\gamma>0$ si $S_{L q}>0$. En notant que $E(q L)=E\left(q \bar{L}_{q}\right)$, on obtient:

$\bar{\Pi}^{r}(q)-\Pi^{u}=2(q-\bar{q}) \bar{L}+\gamma[q(q-\bar{q})-\operatorname{Var}(q)]$.

Comme $E[q(q-\bar{q})]=\operatorname{Var}(q)$, il suffit de choisir $q$ suffisamment grand, avec évidemment $q>\bar{q}$, pour que l'on ait $\Pi^{r}(q)-\Pi^{u}>0$. 


\section{Tableau 1}

Classement moyen des formules de tarification par ordre de préférence entre classes de risque et de dommage

(les classements sont inversés pour $L<<\bar{L}$ et $q<<\bar{q}$ )

\begin{tabular}{cccccc}
\hline & & $\Pi^{u}$ & $\bar{\Pi}^{d}$ & $\bar{\Pi}^{r}$ & $\bar{\Pi}^{m}$ \\
& $S_{L q}=0$ & 1 & 3 & 2 & 3 \\
& $S_{L q}<0$ & $n .^{a} .^{a}$ & 3 & 1 & 2 \\
& $S_{L q}>0$ & 1 & 2 ou 3 & 2 ou 3 & 4 \\
& $S_{L q}=0$ & 1 & 2 & 3 & 3 \\
& $S_{L q}<0$ & $n .^{a} .^{a}$ & 1 & 3 & 2 \\
& $S_{L q}>0$ & 1 & 2 ou 3 & 2 ou 3 & 4 \\
\hline
\end{tabular}

a/ Ne peut être classé

Proposition 3. A populations données de conducteurs, le classement moyen des formules de tarification entre catégories de risque et catégories de dommage a les caractéristiques suivantes:

(i) Sauf peut-être pour les cas où facteurs de risque et dommages sont négativement corrélés, les conducteurs à risque ou à dommage élevé préféreront en moyenne la tarification uniforme à toute autre formule de tarification.

(ii) Sauf peut-être pour le cas où facteurs de risque et dommages sont positivement corrélés, les conducteurs à dommage élevé préféreront en moyenne la tarification sur base de responsabilité à la tarification sur base d'assurance individulle; l'inverse est vrai pour les conducteurs à facteur de risque élevé.

(iii) Dans tous les cas, avec une tarification sur base de responsabilité, les conducteurs à dommage modéré subventionnent en moyenne les conducteurs à dommage élevé par rapport à une tarification au coût marginal; avec une tarification sur base d'assurance individuelle, les conducteurs à facteur de risque modéré subventionnent en moyenne les conducteurs à facteur de risque élevé comparativement à une tarification au coût marginal.

Les résultats présentés ici font abstraction des changements de composition associés à chaque formule de tarification. Comme une tarification au coût marginal pénalise les conducteurs à risque et à dommage élevés, son adoption (en supposant une certaine élasticité de la demande) entraînera une réduction du facteur de risque moyen $\bar{q}$ et du dommage moyen $\bar{L}$ par rapport à une tarification uniforme. Pour les conducteurs dont les caractéristiques de risque ou de dommage sont voisines de la moyenne (ou inférieures), ceci diminuera la facture à payer pour l'accès au réseau (péage uniforme et prime d'assurance proprement dite). L'adoption d'une tarification non uniforme sur base d'assurance individuelle ou de responsabilité aurait un effet semblable, mais moins marqué. 


\section{Conclusion}

La question traitée ici est celle de la tarification d'un système d'assurance obligatoire compensant intégralement les pertes économiques dues aux accidents de la route. On a considéré que seuls les utilisateurs de véhicules automobiles subissaient le coût de ces accidents. L'assurance étant obligatoire, l'accès au réseau routier va de pair avec la participation au système d'assurance. Dans une telle situation, le droit d'accès optimal au réseau routier est la somme d'un péage uniforme représentant la part de chaque conducteur dans le financement de la capacité de ce réseau et d'une prime personnalisée représentant sa part de financement du régime d'assurance. Cette prime d'assurance personnalisée tient compte des caractéristiques de risque du conducteur et du dommage auquel il s'expose en conduisant.

De façon générale, la prime d'assurance optimale diffère de la tarification que l'on retrouve habituellement dans les régimes publics ou privés d'assurance automobile, qu'il s'agisse d'assurance individuelle pure ou impliquant une part de responsabilité civile. Dans des cas particuliers, lorsque par exemple tous les conducteurs ont une propension identique à causer des accidents, une formule d'assurance individuelle pure sera optimale; lorsque tous les conducteurs sont caractérisés par des dommages identiques en cas d'accident,une formule basée sur le principe de responsabilité stricte sera optimale. Dans le cas général, l'assurance individuelle pure implique la présence d'un interfinancement entre automobilistes au détriment des conducteurs à risque modéré; avec une formule basée sur la responsabilité, il y interfinancement au détriment des conducteurs à dommage modéré.

L'analyse présentée ici fournit un cadre pour l'imputation différenciée, entre catégories de conducteurs, du coût social de l'utilisation de l'automobile. On pourrait généraliser ce cadre pour tenir compte de degrés d'exposition au risque différents entre automobilistes (intensité d'utilisation de l'automobile) et de l'influence du comportement volontaire des individus sur le facteur de risque qu'ils représentent (activités de prévention). Bien que cela signifie l'abandon d'une certaine symétrie par rapport à la formulation adoptée ici, il serait possible aussi de généraliser l'analyse au cas des accidents véhicule-piéton (ou véhiculecycliste, etc). L'élément intéressant dans ce cas serait évidemment d'analyser quel jeu d'hypothèses permettrait d'imputer aux seul automobilistes la totalité du coût actuariel des accidents ou, le cas échéant, d'analyser la répartition de ce coût entre automobilistes et piétons ${ }^{11}$.

\section{RÉFÉRENCES}

BOYER, M. et G. DIONNE [1984]: "Sécurité routière: efficacité, subvention et réglementation", L'Actualité Economique, 60 (juin 1984), 200-22.

BOYER, M. et G. DIONNE [1985]: "Sécurité routière: responsabilité pour négligence et tarification", Revue Canadienne d'Economique, 18 (novembre 1985), 814-30.

\footnotetext{
${ }^{11}$ Dans la littérature juridique, notamment celle qui a entouré les discussions sur le no-fault, on a le plus souvent traité comme axiomatique le principe selon lequel "motoring should pay its way".
} 
BOYER, M. et G. DIONNE [1987]: "Description and analysis of the Quebec automobile insurance plan", Canadian Public Policy, 13 (juin 1987), 181-195.

BROWN, J. [1973]: "Toward an economic theory of liability", Journal of Legal Studies, 2 (juin 1973), 323-350.

CNUCED [1987]:L'assurance automobile et l'indemnisation des victimes d'accidents de la circulation dans les pays en voie de développement, Nations Unies, New York.

DAHLBY, B.A. [1983]: "Adverse selection and statistical discrimination", Journal of Public Economics, 20(1983), 121-131.

DIAMOND, P. [1974]: "Single activity accidents", Journal of Legal Studies, 3 (janvier 1974), 107-164.

GREEN, J. [1976]: "On the optimal structure of liability laws", Bell Journal of Economics, 7 (automne 1976), 553-574.

HENRIET, D. et J.-C. ROCHET [1986]: «La logique des systèmes bonus-malus en assurance automobile: une approche théorique», Annales d'Economie et de Statistique, 1 (janvier-mars 1986), 133-152.

HOFFER, G.E. et E.G. MILLER [1979]:"The distribution of automobile liability insurance: an alternative", Journal of Risk and Insurance, 66 (septembre 1979), 441-450.

HOY, M. [1982]: "Categorizing risk in the insurance industry", Quarterly Journal of Economics, 97 (mai 1982), 321-337.

JOHNSON, W.R. [1977]:"Choice of compulsory insurance schemes under adverse selection", Public Choice, 31 (automne 1977), 23-35.

LANDES, E.M. [1982]: "Insurance, liability and accidents: A theoretical and empirical investigation of no-fault accidents", Journal of Law and Economics, 25 (avril 1982),49-65.

OAKLAND, W.H. [1972]:"Congestion, public goods and welfare", Journal of Public Economics, 1 (1972), 339-357.

PAULY, M.V. [1970]: "The welfare economics of community rating", Journal of Risk and Insurance, 37 (septembre 1970), 407-418.

PAULY, M.V. [1974]: "Overinsurance and public provision of insurance", Quarterly Journal of Economics, 88 (printemps 1974), 44-62.

REPORT BY THE AUTOMOBILE ACCIDENT COMPENSATION COMMITTEE OF BRITISHCOLUMBIA [1983], Vancouver.

SHAVELL, S. [1980a]: "Strict liability versus negligence", Journal of Legal Studies, 9 (janvier 1980), $1-25$.

SHAVELL, S. [1980b]: "An analysis of causation and the scope of liability in the law of torts", Journal of Legal Studies, 9 (mars 1980), 463-516.

SHAVELL, S. [1982]:“On liability and insurance”, Bell Journal of Economics, 13 (printemps 1982), $120-132$.

SHAVELL, S. [1985]:"Uncertainty over causation and the determination of civil liability", Journal of Law and Economics, 28 (octobre 1985), 587-608.

TUNC, A. (Ed.) [1981a]: Pour une loi sur les accidents de la circulation, Economica, Paris.

TUNC, A. [1981b]: La responsabilité civile, Economica, Paris.

WILSON, C. [1977]: "A model of insurance markets with incomplete information", Journal of Economic Theory, 16 (décembre 1977), 167-207. 